\title{
Initial Experience of Ramucirumab Treatment After Lenvatinib Failure for Patients With Advanced Hepatocellular Carcinoma
}

\author{
TEIJI KUZUYA, MASATOSHI ISHIGAMI, TAKANORI ITO, YOJI ISHIZU, \\ TAKASHI HONDA, TETSUYA ISHIKAWA and MITSUHIRO FUJISHIRO
}

Department of Gastroenterology and Hepatology, Nagoya University Graduate School of Medicine, Nagoya, Japan

\begin{abstract}
Background/Aim: The outcomes of ramucirumab after lenvatinib failure for hepatocellular carcinoma $(\mathrm{HCC})$ patients with alpha fetoprotein (AFP) levels of $\geq 400 \mathrm{ng} / \mathrm{ml}$ are unknown. Patients and Methods: Of 12 patients treated with ramucirumab after lenvatinib failure, 10 patients were enrolled in this retrospective study. Results: The disease control rate of $80 \%$ at 6 weeks and the median time to progression of 3.1 months were the same by both the Response Evaluation Criteria in Solid Tumors (RECIST) and the modified RECIST. $A F P$ reduction was seen in 5 patients at 2 weeks and in 3 patients at 6 weeks. The incidence of grade 3 adverse events was low at 10\%. The albumin-bilirubin scores within 6 weeks did not worsen. Conclusion: Ramucirumab might have potential therapeutic efficacy and safety in advanced HCC patients after lenvatinib failure. Further studies are needed to confirm the outcomes of ramucirumab after lenvatinib failure.
\end{abstract}

Since 2019, based on the positive results of the REACH-2 trial (1), ramucirumab, which is a human $\operatorname{IgG} 1$ monoclonal antibody that inhibits the ligand activation of vascular endothelial growth factor receptor-2 (2), has become available as an effective second-line therapy following sorafenib treatment in patients with advanced hepatocellular carcinoma (HCC) and baseline alpha fetoprotein (AFP) level of $\geq 400 \mathrm{ng} / \mathrm{ml}$. According to the clinical practice guidelines on the management of $\operatorname{HCC}(3,4)$, both sorafenib and lenvatinib were recommended as the first-line systemic therapy options for patients with advanced stage HCC. Because the REFLECT trial reported superior antitumor

This article is freely accessible online.

Correspondence to: Teiji Kuzuya, Department of Gastroenterology and Hepatology, Nagoya University Graduate School of Medicine, 65 Tsuruma-cho, Showa-ku, Nagoya, Aichi 466-8550, Japan. Tel: +81 527442169, Fax: +81 527442178, e-mail: tkuzuya@med.nagoyau.ac.jp

Key Words: Alpha fetoprotein, hepatocellular carcinoma, lenvatinib, ramucirumab, second-line therapy. response and progression-free survival (PFS) with lenvatinib over sorafenib $(5,6)$, lenvatinib has been increasingly selected as the first-line therapy in clinical practice (7-10). However, the outcomes of ramucirumab treatment after lenvatinib failure are unknown, because the REACH-2 trial was conducted on patients with prior sorafenib intake (1). Accordingly, the aims of this study were to investigate the efficacy and safety of ramucirumab in patients with advanced HCC after lenvatinib failure in clinical practice.

\section{Patients and Methods}

Patients. According to the inclusion criteria of the REACH-2 trial (1), ramucirumab treatment was started in patients with an HCC stage that was equivalent to Barcelona Clinic Liver Cancer stage $\mathrm{C}$ or $\mathrm{B}$ and who were not eligible for surgical resection or locoregional therapy and those with Eastern Cooperative Oncology Group Performance Status (ECOG-PS) score of 0 or 1, Child-Pugh class A, and AFP levels of $\geq 400 \mathrm{ng} / \mathrm{ml}$. Between June 2019 and Jan 2020, 12 consecutive patients with advanced HCC and who were judged by the Response Evaluation Criteria in Solid Tumors (RECIST) as progressive disease (PD) after lenvatinib treatment were initiated on ramucirumab treatment at our hospital. Patients who were observed for $<6$ weeks were excluded. The remaining 10 patients were enrolled in this study and underwent retrospective evaluation of their outcomes. This study was approved by the ethics committee of Nagoya University Graduate School of Medicine (2019-0031) and was performed in compliance with the 1975 Declaration of Helsinki.

Ramucirumab treatment and assessment of adverse events and changes in liver function. Ramucirumab was intravenously injected at a dose of $8 \mathrm{mg} / \mathrm{kg}$ once every 2 weeks. Adverse events (AEs) were assessed according to the Common Terminology Criteria for Adverse Events version 4.0. If drug-related AEs occurred, dose reduction or temporary interruption was done until the symptoms resolved to grade 1 or 2 , according to the guidelines provided by the manufacturer. Ramucirumab treatment was continued until the occurrence of potentially fatal AEs or until clinical tumor progression. As for the assessment of changes in liver function, the albumin-bilirubin (ALBI) score was investigated at baseline and at 2, 4, and 6 weeks.

Evaluation of antitumor and AFP responses. Antitumor responses were evaluated according to both the RECIST and the modified RECIST (mRECIST) $(11,12)$. Four-phase (i.e., unenhanced, late 
arterial, portal venous, and equilibrium) contrast-enhanced computed tomography (CE-CT) examination was performed with a predetermined schedule at baseline and at 6 weeks after ramucirumab initiation and every 4 to 10 weeks thereafter. The analyzed HCC tumor marker was serum AFP at baseline and at 2, 4, and 6 weeks after ramucirumab initiation. For each patient, the baseline concentration of AFP was assigned a value of 1, and the ratios of AFP at 2, 4, and 6 weeks after ramucirumab initiation were calculated. AFP ratios were expressed as medians \pm standard error (SE).

Statistical analysis. Statistical analyses were performed using Easy R (EZR) version 1.29 (Saitama Medical Center, Jichi Medical University, Saitama, Japan) (13). Time to progression (TTP) after ramucirumab initiation was measured from the start date of ramucirumab until the date of confirmation of the first radiologic PD, according to both the RECIST and mRECIST. The treatment duration of ramucirumab was measured from the start date of treatment until the date of discontinuation. TTP and treatment duration were calculated using the Kaplan-Meier method.

\section{Results}

Baseline patient characteristics. Table I shows the baseline characteristics of the $10 \mathrm{HCC}$ patients enrolled in this study. The population comprised 5 men and 5 women, with a median age of 76 years (range $=42-89$ years). There were 7 patients with an ECOG-PS of 0 and 6 patients with a ChildPugh score of 5 . The median AFP level was $3,421 \mathrm{ng} / \mathrm{ml}$ (range $=633-82,429 \mathrm{ng} / \mathrm{ml}$ ). Ramucirumab was initiated as second-line treatment in 8 patients and as fourth-line treatment in 2 patients. The median observation period was 3.6 months (range $=1.8-5.8$ months).

Efficacy. With regard to the antitumor response at 6 weeks according to RECIST, no patient had complete response (CR) and partial response (PR), 8 patients had stable disease (SD), and 2 patients had PD. The objective response rate (ORR) was $0 \%$, and the disease control rate (DCR) was $80 \%$. Concerning antitumor response at 6 weeks according to mRECIST, there were 0 CR, 1 PR, 7 SD and 2 PD designations. The ORR was $10 \%$, and the DCR was $80 \%$. The median TTP of 3.1 months (range=1.4-5.8 months) was the same by both RECIST and mRECIST. As for the AFP changes (Figure 1A), the median AFP ratios at 2, 4, and 6 weeks were 1.01 (range $=0.71-3.20$ ); 1.09 (range $=0.34-3.38$ ); and 1.23 (range=0.27-3.05), respectively. An AFP ratio of $\leq 1.0$ was seen in 5 patients $(50 \%)$ at 2 weeks and in 3 patients $(30 \%)$ at 6 weeks. All 3 patients with reduced AFP at 6 weeks already had reduced AFP at 2 weeks. Three representative cases are shown in Figure 2. Case 1 was judged as SD by both RECIST and mRECIST (Figure 2A and B) and had an AFP level change from $1,993 \mathrm{ng} / \mathrm{ml}$ at baseline to $1,996 \mathrm{ng} / \mathrm{ml}$ at 6 weeks. Case 2 was judged as SD by RECIST and as PR by mRECIST (Figure 2C-F) and had an AFP level change from 82,429 $\mathrm{ng} / \mathrm{ml}$ at baseline to $22,490 \mathrm{ng} / \mathrm{ml}$ at 6 weeks.
Table I. Baseline characteristics of the HCC patients enrolled in this study.

\begin{tabular}{lc}
\hline Characteristics & $\mathrm{n}=10$ \\
\hline Median age (years, range) & $76(42-89)$ \\
Gender (male/female) & $5 / 5$ \\
Etiology (HBV/HCV/NBNC) & $2 / 5 / 3$ \\
ECOG-PS $(0 / 1)$ & $7 / 3$ \\
Child-Pugh score $(5 / 6)^{\dagger}$ & $6 / 3$ \\
mALBI grade $(1 / 2 \mathrm{a} / 2 \mathrm{~b})^{\dagger}$ & $2 / 3 / 4$ \\
BCLC stage (B/C) & $6 / 4$ \\
Tumor size $(<30 \mathrm{~mm} / \geq 30 \mathrm{~mm})$ & $8 / 2$ \\
Number of tumors $(<4 / \geq 4)$ & $2 / 8$ \\
Portal vein tumor thrombosis (absent/present) & $8 / 2$ \\
Extrahepatic spread (absent/present) & $7 / 3$ \\
Median serum AFP level (ng/ml, range) & $3421(633-82429)$ \\
Ramucirumab treatment $\left(2^{\text {nd }}\right.$ line/4th line) & $8 / 2$ \\
Median observation period (months) & $3.6(1.8-5.8)$ \\
\hline
\end{tabular}

HCC: Hepatocellular carcinoma; HBV: hepatitis B virus; HCV: hepatitis $\mathrm{C}$ virus; NBNC: non-HBV and non-HCV; ECOG: Eastern Cooperative Oncology Group; PS: performance status; mALBI: modified albuminbilirubin; BCLC: Barcelona Cancer Liver Clinic; AFP: alpha fetoprotein. †one patient was excluded because she had constitutional jaundice.

Safety. Table II shows the frequency of AEs within 6 weeks after ramucirumab initiation in a total of 10 patients. The most common AEs were hypertension $(n=5)$, appetite loss $(n=4)$, proteinuria $(n=3)$, and generalized fatigue $(n=3)$. Two patients needed ramucirumab dose reduction because of grade 2 appetite loss. The incidence of grade $3 \mathrm{AEs}$ was low at $10 \%$ (i.e., 1 case of grade 3 fever). At 3 weeks, that patient (Case 3) developed fever, and the AFP level changed to $462 \mathrm{ng} / \mathrm{ml}$ from $672 \mathrm{ng} / \mathrm{ml}$ at baseline; at that time, the disappearance of arterial tumor enhancement on CE-CT images was seen (Figure $2 \mathrm{G}$ and $\mathrm{H}$ ). Therefore, because the fever was considered to reflect antitumor response, ramucirumab was administered on schedule immediately after the fever resolved. Grade 4 AEs were not observed. The median treatment duration of ramucirumab was 4.6 months (range $=1.6-5.8$ months). No patient died during the observation period. The changes in the ALBI score within 6 weeks were evaluated in 9 patients but not in 1 patient who had constitutional jaundice (Figure 1B). The median ALBI scores at baseline and at 2, 4, and 6 weeks were $-2.27 \pm 0.37 ;-2.27 \pm 0.39 ;-2.27 \pm 0.47$; and $-2.38 \pm 0.61$, respectively.

\section{Discussion}

Currently, there is no established effective second-line treatment following lenvatinib failure. To our best knowledge, this was the first study that investigated the outcomes of ramucirumab in patients with advanced HCC after lenvatinib failure in clinical practice. 

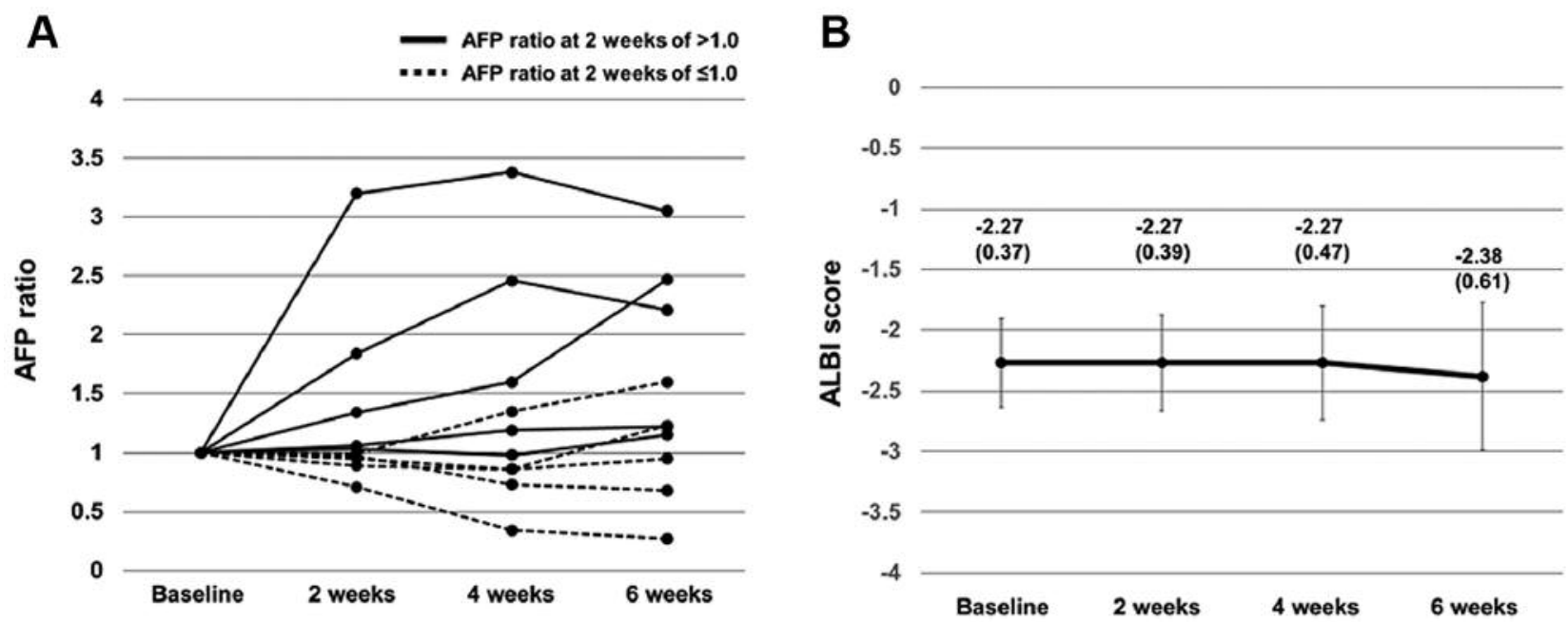

Figure 1. Changes in AFP ratio (A) and ALBI score (B) at 2, 4, and 6 weeks. (A) The median AFP ratios at 2, 4, and 6 weeks are 1.01 (range $=0.71$ 3.20), 1.09 (range =0.34-3.38), and 1.23 (range=0.27-3.05), respectively. An AFP ratio of $\leq 1.0$ is seen in 5 patients (50\%) at 2 weeks and in 3 patients (30\%) at 6 weeks. All 3 patients with reduced AFP at 6 weeks already had reduced AFP at 2 weeks. (B) The median ALBI scores at baseline and at 2,4 , and 6 weeks were $-2.27 \pm 0.37 ;-2.27 \pm 0.39 ;-2.27 \pm 0.47$; and $-2.38 \pm 0.61$, respectively.

AFP: Alpha fetoprotein; ALBI: albumin-bilirubin.

(A) At baseline (Case 1)

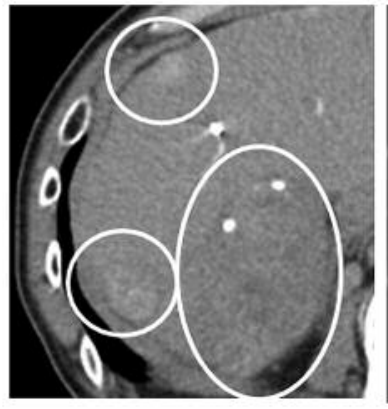

(E) At baseline (Case 2)

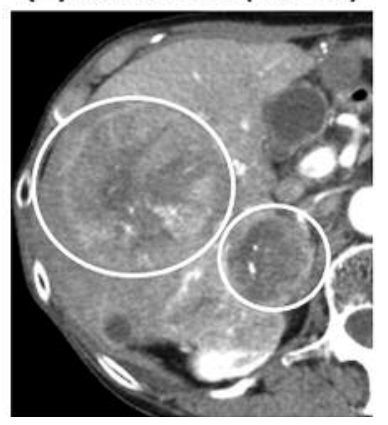

(B) At 6 weeks (Case 1)
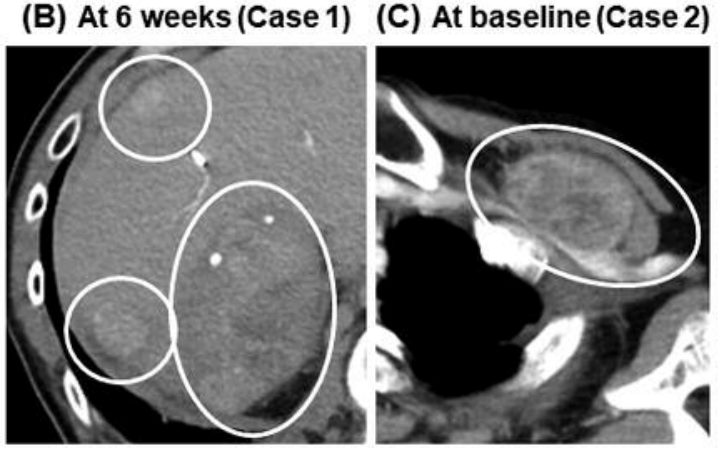

(D) At 6 weeks (Case 2)

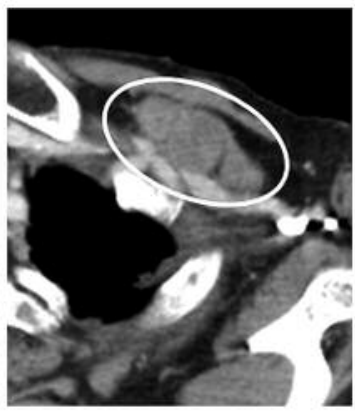

(F) At 6 weeks (Case 2)

(G) At baseline (Case 3) (H) At 3 weeks (Case 3)
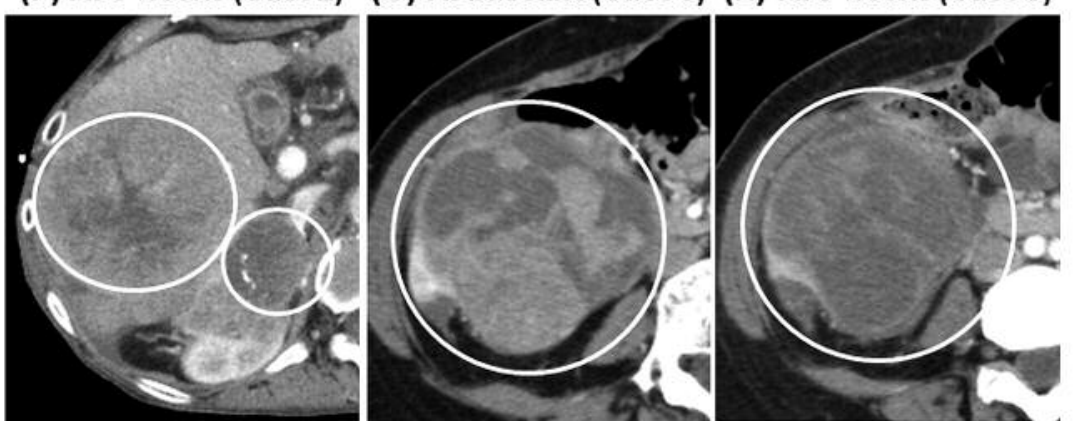

Figure 2. CE-CT images in the late arterial phase of 3 representative cases. (A and B) Case 1, who is judged as SD by both RECIST and $m R E C I S T$ at 6 weeks and whose AFP level changed from 1,993 $\mathrm{ng} / \mathrm{ml}$ at baseline to 1,996 $\mathrm{ng} / \mathrm{ml}$ at 6 weeks. (C-F) Case 2, who is judged as SD by RECIST and as PR by $\mathrm{mRECIST}$ and whose AFP level changed from $82,429 \mathrm{ng} / \mathrm{ml}$ at baseline to 22,490 $\mathrm{ng} / \mathrm{ml}$ at 6 weeks. (G and H) Case 3 developed fever at 3 weeks, when the AFP level changed from $672 \mathrm{ng} / \mathrm{ml}$ at baseline to $462 \mathrm{ng} / \mathrm{ml}$. At this time, the disappearance of arterial tumor enhancement on CE-CT images was seen. CE-CT: Contrast-enhanced computed tomography; SD: stable disease; RECIST: Response Evaluation Criteria in Solid Tumors; mRECIST: modified RECIST; AFP: alpha fetoprotein; PR: partial response. 
Table II. Adverse events of ramucirumab treatment within 6 weeks $(n=10)$.

\begin{tabular}{|c|c|c|c|c|c|}
\hline Adverse events & $\begin{array}{c}\text { Grade } 1 \\
\mathrm{n}(\%)\end{array}$ & $\begin{array}{c}\text { Grade } 2 \\
\text { n }(\%)\end{array}$ & $\begin{array}{c}\text { Grade } 3 \\
\mathrm{n}(\%)\end{array}$ & $\begin{array}{c}\text { Grade } 4 \\
\mathrm{n}(\%)\end{array}$ & $\begin{array}{c}\text { Any grade } \\
\text { n }(\%)\end{array}$ \\
\hline Hypertension & $2(20)$ & $3(30)$ & 0 & 0 & $5(50)$ \\
\hline Appetite loss & $1(20)$ & $3(30)$ & 0 & 0 & $4(40)$ \\
\hline Proteinuria & $2(20)$ & $1(10)$ & 0 & 0 & $3(30)$ \\
\hline Generalized fatigue & $3(30)$ & 0 & 0 & 0 & $3(30)$ \\
\hline Peripheral edema & 0 & $2(20)$ & 0 & 0 & $2(20)$ \\
\hline Fever & 0 & $1(10)$ & $1(10)$ & 0 & $2(20)$ \\
\hline Hypothyroidism & 0 & $2(20)$ & 0 & 0 & $2(20)$ \\
\hline Hand-foot skin reaction & $1(10)$ & 0 & 0 & 0 & $1(10)$ \\
\hline Rash & $1(10)$ & 0 & 0 & 0 & $1(10)$ \\
\hline
\end{tabular}

The REACH-2 trial evaluated antitumor response only by conventional RECIST and reported ORR and DCR of $4.6 \%$ and $59.5 \%$, respectively; the median PFS according to the RECIST was 2.8 months $(95 \% \mathrm{CI}=2.8-4.1$ months $)$ in the ramucirumab group (1). The present study showed RECIST and mRECIST ORRs of $0 \%$ and $10 \%$, respectively, and DCRs of $80 \%$ and $80 \%$, respectively, at 6 weeks. Though some cases (i.e., cases 2 and 3) did not demonstrate changes in the size of the entire tumor but showed the disappearance or decrease of arterial tumor enhancement on CE-CT images, except 1 case, the remaining 9 patients were matched by both criteria. The median TTP of 3.1 months was the same by both the RECIST and mRECIST. Therefore, the judgment of PD was matched according to both the RECIST and mRECIST. Similar to the REACH-2 trial, the judgment of ramucirumab failure based on only the RECIST might be appropriate.

Many investigators have reported that AFP reduction after systemic therapy, such as sorafenib and lenvatinib, was a useful prognostic marker of good outcomes $(8,14)$. The REACH-2 trial was the first to show efficacy with a biomarker AFP level of $\geq 400 \mathrm{ng} / \mathrm{ml}$, it documented that AFP level at baseline was a prognostic factor related to OS, but it did not document AFP response (1). In the present study, AFP reductions at 2 and 6 weeks were seen in $5(50 \%)$ and $3(30 \%)$ patients, respectively, and all 3 patients with reduced AFP at 6 weeks already had reduced AFP at 2 weeks. Moreover, AFP reduction was observed in patients (i.e., cases 2 and 3) with the disappearance or decrease of arterial tumor enhancement on CE-CT images. Our initial experience suggested that early AFP reduction after ramucirumab initiation might reflect good antitumor response.

In the REACH-2 trial, the frequency of serious AEs of any grade and cause was $35 \%$ in the ramucirumab group, whereas that of treatment-related AEs of any grade was $11 \%$. Similarly, in the present study, the incidence of grade 3 AEs within 6 weeks was low at 10\%. Several reports documented deterioration of liver function in the early period after the initiation of sorafenib or lenvatinib (15-17). This present study found that the ALBI scores did not worsen within 6 weeks after ramucirumab initiation. Based on the REACH-2 trial and our results, ramucirumab could be well tolerated.

There were several limitations to the present study. First was the retrospective and nonrandomized design. Second was the small sample size and the short observation period. Therefore, confirmation of our findings would require additional studies on a larger number of patients in an independent cohort.

In conclusion, our results suggested that ramucirumab after lenvatinib failure has potential therapeutic efficacy and safety in patients with advanced HCC and AFP levels of $\geq 400 \mathrm{ng} / \mathrm{ml}$. Further studies are needed to confirm the outcomes of ramucirumab after lenvatinib failure.

\section{Conflicts of Interest}

Teiji Kuzuya received lecture fees from Eisai and Bayer. All other Authors declare no competing interests.

\section{Authors' Contributions}

Conceptualization: Teiji Kuzuya; Methodology: Teiji Kuzuya; Formal analysis and investigation: Teiji Kuzuya and Masatoshi Ishigami; Data curation, Teiji Kuzuya, Masatoshi Ishigami, Takanori Ito, Yoji Ishizu, Takashi Honda and Tetsuya Ishikawa; Writing original draft preparation: Teiji Kuzuya; Writing - review and Editing: Masatoshi Ishigami; Supervision: Mitsuhiro Fujishiro. All Authors approved the final draft of the manuscript.

\section{Acknowledgements}

There was no grant or other financial support for this study.

\section{References}

1 Zhu AX, Kang YK, Yen CJ, Finn RS, Galle PR, Llovet JM, Assenat E, Brandi G, Pracht M, Lim HY, Rau KM, Motomura K, Ohno I, Merle P, Daniele B, Shin DB, Gerken G, Borg C, Hiriart JB, Okusaka T, Morimoto M, Hsu Y, Abada PB, Kudo M and REACH- 
2 study investigators: Ramucirumab after sorafenib in patients with advanced hepatocellular carcinoma and increased $\alpha$-fetoprotein concentrations (REACH-2): a randomised, double-blind, placebocontrolled, phase 3 trial. Lancet Oncol 20(2): 282-296, 2019. PMID: 30665869. DOI: 10.1016/S1470-2045(18)30937-9

2 Spratlin JL, Cohen RB, Eadens M, Gore L, Camidge DR, Diab S, Leong S, O'Bryant C, Chow LQ, Serkova NJ, Meropol NJ, Lewis NL, Chiorean EG, Fox F, Youssoufian H, Rowinsky EK and Eckhardt SG: Phase I pharmacologic and biologic study of ramucirumab (IMC-1121B), a fully human immunoglobulin G1 monoclonal antibody targeting the vascular endothelial growth factor receptor-2. J Clin Oncol 28(5): 780-787, 2010. PMID: 20048182. DOI: $10.1200 / \mathrm{JCO} .2009 .23 .7537$

3 European Association for the Study of the Liver. Electronic address: easloffice@easloffice.eu; European Association for the Study of the Liver: EASL Clinical Practice Guidelines: Management of hepatocellular carcinoma. J Hepatol 69(1): 182236, 2018. PMID: 29628281. DOI: 10.1016/j.jhep.2018.03.019

4 Marrero JA, Kulik LM, Sirlin CB, Zhu AX, Finn RS, Abecassis MM, Roberts LR and Heimbach JK: Diagnosis, Staging, and Management of Hepatocellular Carcinoma: 2018 Practice Guidance by the American Association for the Study of Liver Diseases. Hepatology 68(2): 723-750, 2018. PMID: 29624699. DOI: $10.1002 /$ hep. 29913

5 Kudo M, Finn RS, Qin S, Han KH, Ikeda K, Piscaglia F, Baron A, Park JW, Han G, Jassem J, Blanc JF, Vogel A, Komov D, Evans TRJ, Lopez C, Dutcus C, Guo M, Saito K, Kraljevic S, Tamai T, Ren M and Cheng AL: Lenvatinib versus sorafenib in first-line treatment of patients with unresectable hepatocellular carcinoma: a randomised phase 3 non-inferiority trial. Lancet 391(10126): 1163-1173, 2018. PMID: 29433850. DOI: 10.1016/S0140-6736(18)30207-1

6 Yamashita T, Kudo M, Ikeda K, Izumi N, Tateishi R, Ikeda M, Aikata H, Kawaguchi Y, Wada Y, Numata K, Inaba Y, Kuromatsu R, Kobayashi M, Okusaka T, Tamai T, Kitamura C, Saito K, Haruna K, Okita K and Kumada H: REFLECT-a phase 3 trial comparing efficacy and safety of lenvatinib to sorafenib for the treatment of unresectable hepatocellular carcinoma: an analysis of Japanese subset. J Gastroenterol 55(1): 113-122, 2020. PMID: 31720835. DOI: 10.1007/s00535-019-01642-1

7 Hiraoka A, Kumada T, Kariyama K, Takaguchi K, Itobayashi E, Shimada N, Tajiri K, Tsuji K, Ishikawa T, Ochi H, Hirooka M, Tsutsui A, Shibata H, Tada T, Toyoda H, Nouso K, Joko K, Hiasa Y, Michitaka K; Real-life Practice Experts for HCC (RELPEC) Study Group and the HCC 48 Group (hepatocellular carcinoma experts from 48 clinics in Japan): Therapeutic potential of lenvatinib for unresectable hepatocellular carcinoma in clinical practice: Multicenter analysis. Hepatol Res 49(1): 111-117, 2019. PMID: 30144256. DOI: 10.1111/hepr.13243

8 Kuzuya T, Ishigami M, Ito T, Ishizu Y, Honda T, Ishikawa T and Fujishiro M: Favorable radiological antitumor response at 2 weeks after starting lenvatinib for patients with advanced hepatocellular carcinoma. Hepatol Res, 2019. PMID: 31721363. DOI: $10.1111 /$ hepr.13452

9 Takahashi A, Moriguchi M, Seko Y, Ishikawa H, Yo T, Kimura H, Fujii H, Shima T, Mitsumoto Y, Ishiba H, Takashima H, Nagao Y, Jo M, Arai M, Hara T, Okajima A, Muramatsu A, Morita A, Yoshinami N, Nakajima T, Mitsuyoshi H, Umemura A, Nishikawa $\mathrm{T}$, Yamaguchi K and Itoh Y: Impact of relative dose intensity of early-phase lenvatinib treatment on therapeutic response in hepatocellular carcinoma. Anticancer Res 39(9): 5149-5156, 2019. PMID: 31519627. DOI: 10.21873/anticanres.13710

10 Eso Y, Nakano S, Mishima M, Arasawa S, Iguchi E, Nakamura F, Takeda H, Takai A, Takahashi K, Taura K and Seno H: Dose intensity/body surface area ratio is a novel marker useful for predicting response to lenvatinib against hepatocellular carcinoma. Cancers (Basel) 12(1): E49, 2019. PMID: 31877859. DOI: $10.3390 /$ cancers12010049

11 Eisenhauer EA, Therasse P, Bogaerts J, Schwartz LH, Sargent D, Ford R, Dancey J, Arbuck S, Gwyther S, Mooney M, Rubinstein L, Shankar L, Dodd L, Kaplan R, Lacombe D and Verweij J: New response evaluation criteria in solid tumours: revised RECIST guideline (version 1.1). Eur J Cancer 45(2): 228-247, 2009. PMID: 19097774. DOI: 10.1016/j.ejca.2008.10.026

12 Lencioni $\mathrm{R}$ and Llovet JM: Modified RECIST (mRECIST) assessment for hepatocellular carcinoma. Semin Liver Dis 30(1): 52-60, 2010. PMID: 20175033. DOI: 10.1055/s-0030-1247132

13 Kanda Y: Investigation of the freely available easy-to-use software 'EZR' for medical statistics. Bone Marrow Transplant 48(3): 452458, 2013. PMID: 23208313. DOI: 10.1038/bmt.2012.244

14 Kuzuya T, Asahina Y, Tsuchiya K, Tanaka K, Suzuki Y, Hoshioka T, Tamaki S, Kato T, Yasui Y, Hosokawa T, Ueda K, Nakanishi H, Itakura J, Takahashi Y, Kurosaki M and Izumi N: Early decrease in $\alpha$-fetoprotein, but not des- $\gamma$-carboxy prothrombin, predicts sorafenib efficacy in patients with advanced hepatocellular carcinoma. Oncology 81(3-4): 251-258, 2011. PMID: 22116493. DOI: 10.1159/000334454

15 Terashima T, Yamashita T, Sunagozaka H, Arai K, Kawaguchi K, Kitamura K, Yamashita T, Sakai Y, Mizukoshi E, Honda M and Kaneko S: Analysis of the liver functional reserve of patients with advanced hepatocellular carcinoma undergoing sorafenib treatment: Prospects for regorafenib therapy. Hepatol Res 48(12): 956-966, 2018. PMID: 29845710. DOI: 10.1111/hepr.13196

16 Ueshima K, Nishida N, Hagiwara S, Aoki T, Minami T, Chishina H, Takita M, Minami Y, Ida H, Takenaka M, Sakurai T, Watanabe T, Morita M, Ogawa C, Hiraoka A, Johnson P and Kudo M: Impact of baseline ALBI grade on the outcomes of hepatocellular carcinoma patients treated with lenvatinib: A multicenter study. Cancers (Basel) 11(7): E952, 2019. PMID: 31284682. DOI: $10.3390 /$ cancers 11070952

17 Hiraoka A, Kumada T, Atsukawa M, Hirooka M, Tsuji K, Ishikawa T, Takaguchi K, Kariyama K, Itobayashi E, Tajiri K, Shimada N, Shibata H, Ochi H, Tada T, Toyoda H, Nouso K, Tsutsui A, Nagano T, Itokawa N, Hayama K, Imai M, Joko K, Koizumi Y, Hiasa Y, Michitaka K; On behalf of the Real-Life Practice Experts for HCC (RELPEC) Study Group and HCC 48 Group (hepatocellular carcinoma experts from 48 clinics in Japan): Early relative change in hepatic function with lenvatinib for unresectable hepatocellular carcinoma. Oncology 97(6): 334340, 2019. PMID: 31466068. DOI: 10.1159/000502095
Received February 16, 2020

Revised February 26, 2020

Accepted February 27, 2020 